\title{
Tenascin-C Is an Inhibitory Boundary Molecule in the Developing Olfactory Bulb
}

\author{
Helen B. Treloar, ${ }^{1}$ Arundhati Ray, ${ }^{1}$ Lu Anne Dinglasan, ${ }^{1}$ Melitta Schachner, ${ }^{3,4}$ and Charles A. Greer ${ }^{1,2}$ \\ Departments of ${ }^{1}$ Neurosurgery and ${ }^{2}$ Neurobiology, Yale University School of Medicine, New Haven, Connecticut 06520-8082, ${ }^{3}$ Zentrum für Molekulare \\ Neurobiologie, Universitätskrankenhaus Hamburg-Eppendorf, D-20246 Hamburg, Germany, and ${ }^{4}$ Keck Center for Collaborative Neuroscience and \\ Department of Cell Biology and Neuroscience, Rutgers University, Piscataway, New Jersey 08901
}

We recently described the boundary-like expression pattern of the extracellular matrix molecule tenascin-C (Tnc) in the developing mouse olfactory bulb (OB) (Shay et al., 2008). In the present study, we test the hypothesis that Tncinhibits olfactory sensory neuron (OSN) axon growth in the developing $\mathrm{OB}$ before glomerulogenesis. The period of time before glomerular formation begins, when axons remain restricted to the developing olfactory nerve layer $(\mathrm{ONL})$, is crucial for axon sorting. Here, we show with in vitro analyses that OSN neurite outgrowth is inhibited by Tnc in a dose-dependent manner and that, in stripe assays, axons preferentially avoid Tnc. Using Tnc-null mice, we also show that that glomerular development is delayed in the absence of Tnc. In wild-type mice, OSN axons coalesce into immature or protoglomeruli, which further differentiate and segregate into glomeruli. Glomeruli are first identifiable as discrete structures at birth. In null mice, glomeruli appear immature at birth, remain fused to the $\mathrm{ONL}$, and have a significantly larger diameter compared with wild-type controls. By postnatal day 4, null glomeruli are indistinguishable from controls. Thus, OSN axons appear delayed in their coalescence into glomerular structures. These data correlate with behavioral reports of Tnc-null mice, which are delayed by $24 \mathrm{~h}$ in their acquisition of an olfactory behavior (de Chevigny et al., 2006). Collectively, these data demonstrate that Tnc is an inhibitory boundary molecule in the developing $\mathrm{OB}$ during a key period of development.

\section{Introduction}

The projection of axons from the olfactory epithelium (OE) to the olfactory bulb $(\mathrm{OB})$ is topographic. Olfactory sensory neurons (OSNs) express a single odor receptor (OR) from a family of $\sim 1200$. The convergence of OSN axons expressing the same OR from widely distributed OSNs in the OE to discrete glomerular targets in the $\mathrm{OB}$ is a fundamental principle of organization in the olfactory system. This topographic projection appears very specific and is reproducible across animals. The OR proteins are necessary for correct OSN convergence (Mombaerts et al., 1996; Wang et al., 1998); however, it is equally evident that they are alone insufficient for correct targeting. Some guidance molecules are also implicated in targeting (Cutforth et al., 2003; Schwarting et al., 2004; Serizawa et al., 2006; Kaneko-Goto et al., 2008) as well as cAMP signaling from the OR (Imai et al., 2006; Chesler et al., 2007).

We previously described the development of the OSN pathway (Treloar et al., 1996b, 1999). Of note, OSNs undergo a waiting period in the developing $\mathrm{OB}$, in which they remain restricted to the olfactory nerve layer for up to $4 \mathrm{~d}$ before penetrating into

Received May 19, 2009; accepted June 23, 2009

This work was supported in part by National Institutes of Health (NIH) Grants DC005706 and DC007600 (H.B.T.), Howard Hughes Medical Institute and NIH-National Heart, Lung, and Blood Institute fellowships (L.A.D.), Deutsche Forschungsgemeinschaft (M.S.), and NIH Grant DC00210 (C.A.G.). We express thanks to Christine Kaliszewski, Mike Akins, Diego Rodrigez Gil, and Dolores Montoya for technical help, and Janice Mitchell for administrative support. Correspondence should be addressed to Dr. Helen B. Treloar, Department of Neurosurgery, Yale University School of Medicine, P.0. Box 208082, 333 Cedar Street, New Haven, CT 06520-8082. E-mail: helen.treloar@yale.edu. DOI:10.1523/JNEUROSCI.2356-09.2009

Copyright $\odot 2009$ Society for Neuroscience $\quad 0270-6474 / 09 / 299405-12 \$ 15.00 / 0$ deeper layers and forming synapses with the OB projection neurons. This waiting period is likely critical for sorting of axons and establishment of the appropriate topography between $\mathrm{OE}$ and $\mathrm{OB}$. We previously identified tenascin-C (Tnc) in the mouse as a candidate inhibitory boundary molecule in the developing mouse $\mathrm{OB}$, preventing OSN axons from entering deeper layers of the OB during this critical sorting period (Shay et al., 2008).

Here, our aim was to functionally test this hypothesis. Using an in vitro OSN neurite outgrowth assay, we demonstrate that Tnc inhibits OSN neurite outgrowth from OE explants in a dosedependent manner. Moreover, this effect is specific to Tnc. When grown on a different tenascin substrate, tenascin-R (Tnr), which can also be inhibitory to neurite outgrowth (Taylor et al., 1993), there was no effect on outgrowth from the OE explants.

To further characterize the functional significance of Tnc, we examined olfactory development in Tnc-null mice. In Tnc nulls, we found a delay in glomerular formation. At birth, glomerular structures appear immature; they are not separated from the underlying olfactory nerve layer (ONL) and appear "hairy" and larger than wild-type controls. Analysis of glomerular diameter reveals they are significantly larger than controls. By postnatal day 4, however, null glomeruli are indistinguishable in size and appearance from controls, suggesting that axon coalescence and glomerulogenesis are delayed, but not completely blocked, by the absence of Tnc. This delay in glomerular formation is consistent with reports of delayed acquisition of a neonatal olfactory behavior in Tnc nulls (de Chevigny et al., 2006). Collectively, our studies demonstrate that Tnc is important for glomerulogenesis, providing an inhibitory cue for OSN axons in the OB 
Table 1. Antibodies used in this study

\begin{tabular}{|c|c|c|c|}
\hline Primary antibody (catalog \#) & Source of primary; dilution & Secondary antibody & Source of secondary; dilution \\
\hline Pab VGlut2 (AB5907) & Millipore; 1:2500 & Goat anti-guinea pig-488 & Invitrogen; 1:1000 \\
\hline DAPI & Invitrogen; 1:1000 & $\mathrm{N} / \mathrm{A}$ & $\mathrm{N} / \mathrm{A}$ \\
\hline Draq5 & Biostatus, Ltd.; 1:1000 & $\mathrm{N} / \mathrm{A}$ & $\mathrm{N} / \mathrm{A}$ \\
\hline DBA- biotin (B-1035) & Vector Laboratories; 1:50 & Avidin-568 & Vector Laboratories; 1:1000 \\
\hline \multirow[t]{2}{*}{ Pab Tnc (AB19013; lot \#24010549) } & Millipore; 1:200 (IF) 1:1000 (WB) & Donkey anti-rabbit-555 (IF) & Invitrogen; 1:1000 (IF) \\
\hline & & Donkey anti-rabbit-HRP (WB) & Jackson ImmunoResearch; 1:1000 (WB) \\
\hline Mab Tnc clone MTn-12 (T3413) & Sigma-Aldrich; 1:1000 & Donkey anti-rat-HRP (WB) & Jackson ImmunoResearch; 1:1000 (WB) \\
\hline $\operatorname{Mab} \beta$-actin $(A C-15)$ & Abcam; 1:1000 & Goat anti-mouse lgG-HRP & Jackson ImmunoResearch; 1:1000 \\
\hline Pab NCAM (AB5032) & Millipore; 1:1000 & Donkey anti-rabbit-555 & Invitrogen; 1:1000 \\
\hline Mab phosphacan (MAB5210) & Millipore; 1:500 & Goat anti-mouse lgM-555 & Invitrogen; 1:1000 \\
\hline
\end{tabular}

Abbreviations: IF, Immunofluoresence; WB, Western blot; Pab, polyclonal antibody; Mab, monoclonal antibody.

before the period of synapse formation. When the cue is removed, axon coalescence and circuit establishment is delayed, leading to altered olfactory behavior.

\section{Materials and Methods}

PCR and Western blot analysis

Animals. Pregnant, time-mated CD-1 mice (Charles River) were killed with $\mathrm{CO}_{2}$. Embryos ( $n=12-36$ at each age) were collected by cesarean section on gestational days 13,15, and 17 (day of positive vaginal plug was designated day 0 ) and immediately decapitated. Postnatal mice [postnatal day 0 (PND0), PND2, and PND4 ( $n=6-12$ at each age)] were rapidly decapitated. Older postnatal mice (PND7, PND14, and PND21) and adult mice ( $n=3-6$ at each age) were killed with $\mathrm{CO}_{2}$. OBs were dissected and immediately frozen in dry ice. Animal protocols were reviewed and approved by the Yale Animal Care and Use Committee.

$R N A$ extraction and reverse transcription-PCR. RNA was extracted using RNeasy Plus Mini kit (QIAGEN) following the manufacturer's instructions. Briefly, 20-30 mg of frozen OB tissue was homogenized in Buffer RLT Plus with $\beta$-mercaptoethanol. DNA was removed through gDNA Eliminator column, and extracts were washed with $70 \%$ ethanol in RNeasy spin column. After several washes with Buffer RW1 and Buffer RPE, RNA was eluted with water. Total RNA concentrations were determined using spectrophotometry. cDNA was produced for each RNA sample using iScript cDNA Synthesis kit (Bio-Rad Laboratories; catalog $\# 170$-8891) following the manufacturer's instructions. Briefly, $1 \mu \mathrm{g}$ of total RNA was incubated with a mixture of oligo-dT and random hexamers together with the enzyme mix and synthesis kit buffer in a thermocycler programmed for $5 \mathrm{~min}$ at $25^{\circ} \mathrm{C}$ and $30 \mathrm{~min}$ at $42^{\circ}$.

PCR was performed for each primer pair using cDNA from embryonic day 13 (E13), E15, E17, PND0, PND2, and PND4 OB cDNA using Platinum BluePCR SuperMix (Invitrogen). A volume of $0.5 \mu \mathrm{l}$ of $20 \mu \mathrm{M}$ premixed forward and reverse primers (see sequences below) and $2 \mu \mathrm{l}$ of cDNA reactions (see above) were mixed with $45 \mu \mathrm{l}$ of Platinum BluePCR SuperMix, which was activated at $94^{\circ} \mathrm{C}$ for $1 \mathrm{~min}$. cDNA was denatured for $45 \mathrm{~s}$ at $94^{\circ} \mathrm{C}$, annealed for $45 \mathrm{~s}$ at $55^{\circ} \mathrm{C}$, and extended at $72^{\circ} \mathrm{C}$ for $45 \mathrm{~s}$; this cycle was repeated 30 times. Final extension was performed at $72^{\circ} \mathrm{C}$ for $10 \mathrm{~min}$. Primers were as follows $\left(5^{\prime}-3^{\prime}\right)$ : TNC-FWD, GCAACCAAGGACAATGTGTG; TNC-REV, TGTGGTTTCAGACACCCGTA (amplicon, 1844 bp); $\beta$-actin-FWD, GTGGGCCGCTCTAGGCACCAA; $\beta$-actin-REV, CTCTTTGATGTCACGCACGATTTC (amplicon, 540 bp). TNC5fwd, TNC6rev, TNC5S, and TNC6AS primer sequences were synthesized as described previously (Joester and Faissner, 1999).

Immunoblotting. Dissected OBs from E15, E17, PND0, PND2, PND4, PND7, PND14, PND21, and adult mice were homogenized in lysis buffer [0.5 M Tris, pH 6.8, 2\% SDS, and $1 \times$ Complete Protease Inhibitor Mixture (Roche)] using a Dounce homogenizer. Protein concentrations were determined using Pierce BCA Protein Assay kit (Thermo Fisher Scientific; catalog \#23227). Ten micrograms of each protein sample were resolved using 3-8\% Tris-acetate reducing gel (Invitrogen) to separate larger molecular weight proteins. After electrophoresis, proteins were transferred to a nitocellulose membrane with an iBlot dry blotting system (Invitrogen) using program 3, with an 8 min transfer time recommended for larger molecular weight proteins.
Membranes were blocked in 5\% bovine serum albumin (BSA) in Trisbuffered saline (TBS), pH 7.4, and 0.1\% Tween 20 (TBSTw) for $30 \mathrm{~min}$ at room temperature (RT), incubated in 5\% BSA in TBSTw plus primary antibody [either rabbit anti-Tnc (1:1000; Millipore; catalog \#AB19013, lot \#24010549) (note that more recent lot numbers indicate that the antibody was not suitable for immunoblotting, but similar results were obtained with Sigma-Aldrich monoclonal anti-TNC; catalog \#T3413) (see supplemental Fig. 1, available at www.jneurosci.org as supplemental material) or mouse anti- $\beta$-actin after stripping (1:1000; Abcam; catalog \#AC-15)] for $60 \mathrm{~min}$ at RT. Blots were washed three times for 5 min each time in TBSTw, incubated in HRP-conjugated secondary antibodies (donkey anti-rabbit and donkey anti-mouse IgG, respectively; Jackson ImmunoResearch) diluted 1:1000 in TBSTw for 30 min at RT. Blots were washed two times for 5 min each time in TBSTw and one time in TBS before incubation in chemiluminescence buffer (ECL Western blotting reagents; GE Healthcare) for $1 \mathrm{~min}$ at RT. Blots were exposed to Amersham Hyperfilm MP autoradiography film (GE Healthcare). Blots were stripped using Restore Western blot stripping buffer, $15 \mathrm{~min}$ at RT (Thermo Fisher Scientific), washed three times in TBS before incubating with second primary antibody. Blots were digitized using an Epson flatbed scanner. Band size was determined using Molecular Imaging software, version 4.0 (Kodak).

\section{Immunohistochemistry}

Animals. Pregnant, time-mated CD-1 mice (Charles River) were killed with $\mathrm{CO}_{2}$, and embryos were collected by cesarean section at E13, E15, and E17 ( $n=6-9$ at each age). Postnatal mice at PND0, PND2, and PND4 were rapidly decapitated (postnatal days 0,2 , and $4 ; n=4-6$ at each age). Heads were immersion fixed in $4 \%$ paraformaldehyde (PFA) in PBS, $\mathrm{pH} 7.4$, at $4^{\circ} \mathrm{C}$ overnight. After fixation, tissue was washed in PBS for at least $2 \mathrm{~h}$, cryoprotected in $30 \%$ sucrose in PBS at $4^{\circ} \mathrm{C}$, embedded in OCT compound (Tissue-Tek; Bayer Corporation), and snap frozen in a slurry of dry ice and ethanol. Animal protocols were reviewed and approved by the Yale Animal Care and Use Committee.

Cryosectioning. Tissue was serially sectioned at $20 \mu \mathrm{m}$ in the coronal plane using a Reichart-Jung 2800 Frigocut E cryostat. Sections were thaw-mounted onto Superfrost Plus microscope slides (Thermo Fisher Scientific), air dried, and stored at $-20^{\circ} \mathrm{C}$ until use.

Immunohistochemistry. Immunostaining was performed as previously described (Shay et al., 2008). Briefly, sections were blocked with 2\% BSA (Sigma-Aldrich) in TBS containing 0.3\% Triton X-100 (TBST) for 30 min at RT, and then incubated with different combinations of primary antibodies (detailed below; including sources) as a mixture in block solution overnight at $4^{\circ} \mathrm{C}$. Sections were rinsed three times in TBST, incubated in a mixture of corresponding fluorescence-labeled secondary antibodies (detailed below) diluted 1:1000 for $1 \mathrm{~h}$ at RT, rinsed three times in TBST and one time in TBS, and coverslipped in NPG fluorescent mounting media ( $5 \% n$-propyl gallate in glycerol and $25 \mathrm{~mm} \mathrm{NaHCO}_{3}$ ). If needed, fluorescent DNA dye Draq5 (1:1000; Biostatus) or $4^{\prime}, 6^{\prime}$ diamidino-2-phenylindole (DAPI) (1:1000; Invitrogen) was included with secondary antibodies to counterstain nuclei.

Table 1 details the antibodies used in this study. 


\section{Tissue culture}

Substrate preparation. Microexplants of $\mathrm{OE}$ were cultured on poly-Dlysine (PDL) (molecular weight, 30,000-70,000; Sigma-Aldrich; catalog \#P-7886)-coated $\left(50 \mu \mathrm{g} / \mathrm{ml}\right.$ in $\mathrm{dH}_{2} \mathrm{O} ; 30 \mathrm{~min}$ at $37^{\circ} \mathrm{C}$ and $5 \% \mathrm{CO}_{2}$; followed by three rinses in $\mathrm{dH}_{2} \mathrm{O}$ ) eight-well CultureWell chambered cover glasses (Grace Biolabs; catalog \#CWCS 8R-1.0). Cover glasses were subsequently either left uncoated (i.e., $\mathrm{PDL}$ alone) or coated with either EHS-laminin $\left(20 \mu \mathrm{g} / \mathrm{ml}\right.$ in $\mathrm{dH}_{2} \mathrm{O}$, overnight at $37^{\circ} \mathrm{C}$ and $5 \% \mathrm{CO}_{2}$, followed by three rinses in $\mathrm{dH}_{2} \mathrm{O}$; Invitrogen; catalog \#23017-015); Tnc (10, 15,20 , or $50 \mu \mathrm{g} / \mathrm{ml}$ in $\mathrm{dH}_{2} \mathrm{O}$, overnight at $37^{\circ} \mathrm{C}$ and $5 \% \mathrm{CO}_{2}$, followed by three rinses in $\mathrm{dH}_{2} \mathrm{O}$; Millipore; catalog \#CC115); or Tnr $(50 \mu \mathrm{g} / \mathrm{ml}$ in $\mathrm{dH}_{2} \mathrm{O}$, overnight at $37^{\circ} \mathrm{C}$ and $5 \% \mathrm{CO}_{2}$, followed by three rinses in $\mathrm{dH}_{2} \mathrm{O}$; Millipore; catalog \#CC116). It should be noted here that, although purified chick Tnc was used, Bartsch et al. (1995) have shown that both chick retinal ganglion cells and mouse small cerebellar neurons responded equivalently to tenascin purified from either chick or mouse.

Stripe substrate preparation. Striped substrates were created using silicone matrices obtained from the Bonhoeffer Laboratory (Knöll et al., 2007). Briefly, matrices were applied to PDL-coated $(50 \mu \mathrm{g} / \mathrm{ml})$ coverglasses (coated as above), and Tnc $\left(20 \mu \mathrm{g} / \mathrm{ml}\right.$ in $\left.\mathrm{dH}_{2} \mathrm{O}\right)$ was applied to the channels using a syringe. After $1 \mathrm{~h}$ incubation at $37^{\circ} \mathrm{C}$ and $5 \% \mathrm{CO}_{2}$, channels were flushed with three rinses in $\mathrm{dH}_{2} \mathrm{O}$ while aspirating the outlet hole via vacuum. Matrices were removed, a surrounding hydrophobic barrier quickly applied, and $\mathrm{dH}_{2} \mathrm{O}$ reapplied to stripes temporarily to store the substrate before plating explants so extracellular matrix (ECM) proteins did not dry out.

Microexplant preparation. OE explants were harvested from PND1PND3 CD1 pups. Six pups were rapidly decapitated and the OE lining the nasal septum from the dorsal recess of the nasal cavity was dissected in sterile Dulbecco's PBS. Microexplants were prepared by using a brief ( $5 \mathrm{~min}$ ) enzymatic digestion step followed by gentle trituration. Dissected OEs were digested using the Papain Dissociation kit (Worthington Biochemicals) following the manufacturer's instructions. Briefly, components of the dissociation medium were reconstituted per the manufacturer's instructions; tissue was added and the mixture was equilibrated with $95 \% \mathrm{O}_{2} / 5 \% \mathrm{CO}_{2}$. Tissue was dissociated by incubation with activated papain at $37^{\circ} \mathrm{C}$ for $5 \mathrm{~min}$, followed by gentle trituration. Dissociated cells were pelleted and then resuspended in medium containing the papain inhibitor ovomucoid, as provided in the Papain Dissociation kit. Intact cells were separated from cell membranes by centrifuging through a single-step discontinuous density gradient, and the pellet was finally resuspended in culture medium (Neurobasal supplemented with B27, L-glutamine, and penicillin/streptomycin; Invitrogen). Microexplants were then selected using a dissecting microscope in $50 \mu \mathrm{l}$ of media and plated into culture wells. Explants were cultured for $48 \mathrm{~h}$ at $37^{\circ} \mathrm{C}$ and $5 \%$ $\mathrm{CO}_{2}$ before being fixed in $4 \% \mathrm{PFA} / 4 \%$ sucrose in PBS for $30 \mathrm{~min}$ at room temperature followed by three rinses in PBS.

Analysis of neurite outgrowth. Cultures were immunostained and analyzed with MetaMorph image analysis software (Molecular Devices). Briefly, explants were blocked with 2\% BSA in TBST for 30 min, and then incubated rabbit polyclonal neural cell adhesion molecule (NCAM) antibody (Millipore; catalog \#AB5032) for $1 \mathrm{~h}$ at RT, rinsed three times in TBST, incubated in donkey anti-rabbit Alexa 555-conjugated secondary antibody (1:1000; Invitrogen) together with DAPI (1:1000) for $30 \mathrm{~min}$ at $\mathrm{RT}$, rinsed three times in TBST and one time in TBS, and coverslipped in NPG mounting media. Staining was visualized using an Olympus BX51 fluorescence microscope. Analysis was performed with MetaMorph software (Molecular Devices), which measured explant area (area stained by DAPI) and total explant and neurite area (area stained by NCAM) through its Integrated Morphometry Analysis program. Data were graphed and statistically analyzed using one-way ANOVA with Dunnett's multiple-comparison post hoc test with Prism 4 software (GraphPad Software).

\section{Analysis of Tnc-null mice}

Animals. Pregnant, time-mated $\mathrm{Tnc}^{+/+}$and $\mathrm{Tnc}^{-/-}$mice (Evers et al., 2002) were killed, and embryos were collected by cesarean section at E17 ( $n=4$ at each age). Early postnatal mice at PND0, PND2, and PND4 were rapidly decapitated. Heads were immersion fixed in $4 \%$ PFA in PBS.
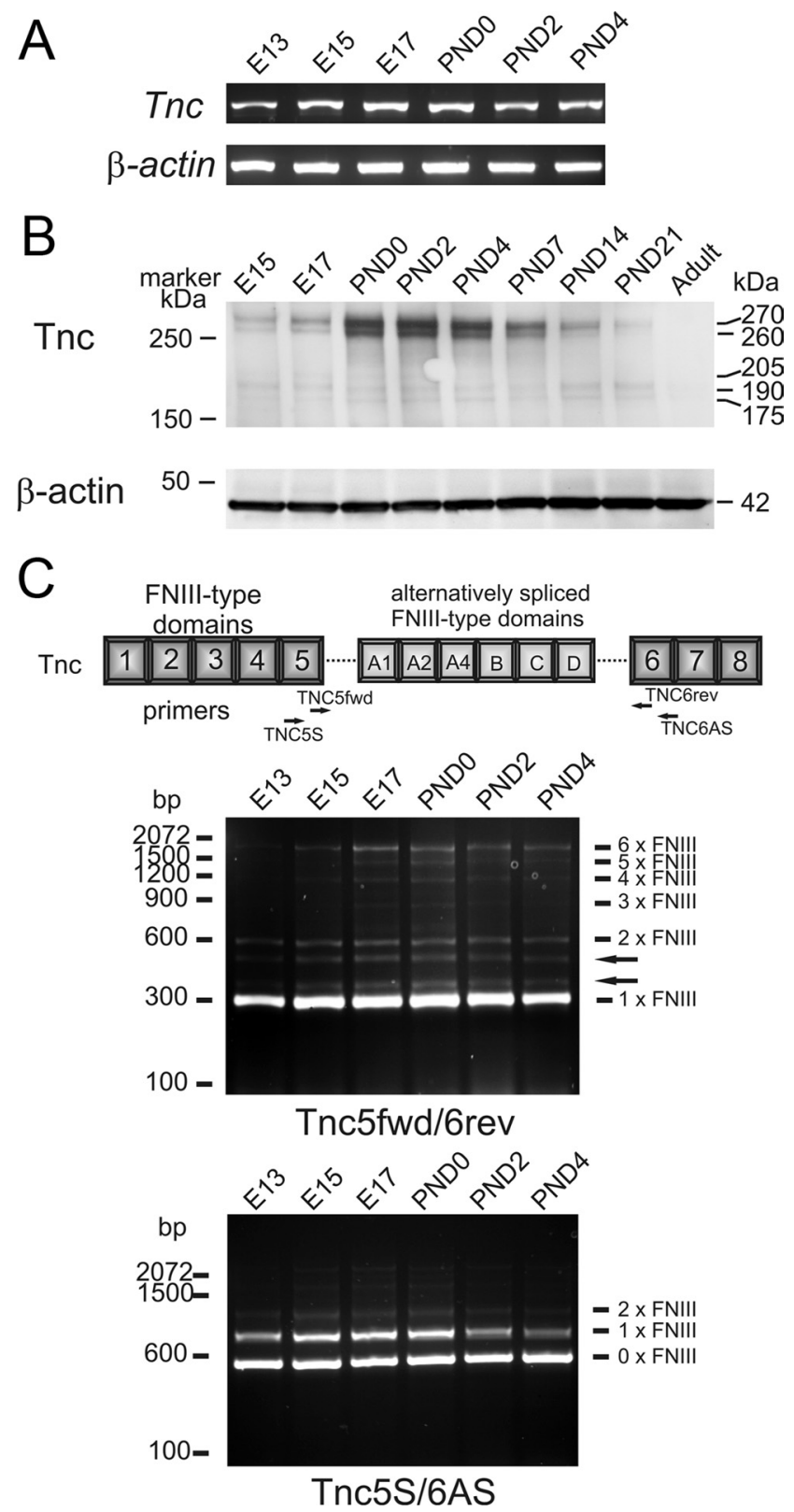

Figure 1. Characterization of Tnc expression in developing olfactory bulb. A, RT-PCR using primers specific for $T n$ and $\beta$-actin using cDNA reverse transcribed from RNA isolated from OBs of various developmental ages. A $1844 \mathrm{bp}$ Tnc amplicon and a $540 \mathrm{bp} \beta$-actin control amplicon product are detected in the $\mathrm{OB}$ at all ages examined. $\boldsymbol{B}$, Western blot analysis of Tnc expression reveals five splice variants of $\sim 175,190,205,260$, and $270 \mathrm{kDa}$ in the OB. Tnc expression of the higher $M_{\mathrm{r}}$ isoforms is found to increase from E15 to birth, when expression peaks. During the postnatal period (birth to P21), expression of these isoforms is observed to decrease. No expression of any isoform is detected in the adult ( $>3$ months). $\beta$-Actin load control ( $42 \mathrm{kDa}$ ) confirms quantity of protein loaded on gel. $C, P C R$ analyses of isoform expression. Using primers that span the alternatively spliced domains, isoforms of all expected sizes are observed (i.e., 0 to 6 FNIII domains). Note the two bands indicated with arrows seen using primer pairs TNC5fwd/ TNC6rev. Although these bands do not correspond to $300 \mathrm{bp} \mathrm{FNIII} \mathrm{domains,} \mathrm{they} \mathrm{have} \mathrm{been}$ previously observed (von Holst et al., 2007) and may represent variant domains in this region.

Tissue was washed in PBS for $24 \mathrm{~h}$, cryoprotected in $30 \%$ sucrose in PBS at $4^{\circ} \mathrm{C}$ until the tissue sank, embedded in OCT compound, and snap frozen in a slurry of dry ice and ethanol.

Cryosectioning. Tissue was serially sectioned at $20 \mu \mathrm{m}$ in the coronal plane using a Reichart-Jung 2800 Frigocut E cryostat. Sections were thaw-mounted onto Superfrost Plus microscope slides (Thermo Fisher Scientific), air dried, and stored at $-20^{\circ} \mathrm{C}$ until use. 
Immunohistochemistry. Immunostaining was performed as above. To assess glomerular formation, every 10th section from rostral to caudal was stained with rabbit anti-NCAM (1: 1000) and guinea pig anti-vesicular glutamate transporter 2 (VGlut2) primary antibodies, followed by donkey anti-rabbit-Alexa 555 and goat anti-guinea pig 488 (both 1:1000) secondary antibodies together with DAPI (1:1000). To assess formation of topographic projections, every 10th section from rostral to caudal was stained with rabbit anti-NCAM (1:1000) and the plant lectin Dolichos biflorus agglutinin (DBA) conjugated to biotin (1:50; Vector Laboratories) followed by donkey antirabbit-Alexa 488 and avidin-568 (both 1:1000) secondary antibodies.

Glomerular diameter analysis. Highmagnification images of the glomerular layer on the medial surface of the $\mathrm{OB}$ were obtained from both the left and right OBs from each of three animals at every age (wild type and null), except for PND4-null mice, in which only two animals were available. Glomerular diameter (parallel to the plane of the nerve layer) was measured in postnatal animals using the caliper function in MetaMorph. Glomerular boundaries were defined using three independent measures: NCAM immunoreactivity, VGlut2 synaptic staining, and DAPI boundaries. Data were graphed and statistically analyzed using Student's $t$ test with Prism 4 software (GraphPad).

\section{Results}

Expression of tenascins in the developing $\mathrm{OB}$

Previous results from our laboratory demonstrated immunolocalization of Tnc in the mouse OB in a boundary-like pattern (Shay et al., 2008). Tnc has been reported to be inhibitory to neurite outgrowth for some classes of neurons or when presented as a sharp substrate boundary with a conducive substrate. To molecularly characterize Tnc expression in the OB, we first used reverse transcription (RT)PCR to examine expression in the developing OB. As seen in Figure 1, using primers to a conserved region of the protein, Tnc mRNA (Fig. $1 A$ ) is detected in the developing $\mathrm{OB}$ during the period of olfactory pathway formation, confirming our previous immunolocalization studies (Shay et al., 2008). To assess changes in protein expression levels during $\mathrm{OB}$ development, we performed a Western blot (Fig. $1 B$ ). Tnc, although encoded by a single gene, exists as a family of alternatively spliced isoforms (Meiners and Geller 1997; Joester and Faissner, 1999; Mercado et al., 2004). Multiple isoforms were observed in the developing OB at $175,190,205,260$, and $270 \mathrm{kDa}$. The predominant species were the $260-270 \mathrm{kDa}$ bands, which increased during the embryonic period and peaked at birth, a time point when glomeruli are first identifiable. Expression was then observed to gradually decrease over the first postnatal week, show a marked decrease over the second postnatal week, and by the end of the third postnatal week have levels of expression lower than E15, the first embryonic age examined. These data are consistent with immunolocalization of Tnc in tissue sections (cf. Shay et al., 2008) (see Fig. 8).

To further investigate the isoforms of Tnc present in the developing $\mathrm{OB}$, we used the PCR-based approach developed by Joester and Faissner (1999) to confirm the observations seen in
Western analyses (Fig. 1C). Using two different primers pairs that span the alternatively spliced domains, these analyses reveal Tnc isoforms of all the predicted sizes present in the developing OB. These data confirm the presence of multiple isoforms seen in Western analyses and indicate differential expression, but do not allow the identification of which specific isoforms are present. It must also be noted that this analysis does not indicate relative abundance of different size isoforms either, as the PCR is biased toward the production of smaller amplicons.

Collectively, these data establish that Tnc upregulates expression during the period of initial olfactory pathway formation and decreases in expression as the pathway is established. Moreover, Tnc has dynamic changes in isoform expression. Thus, Tnc is both spatially and temporally located in the developing olfactory pathway to influence OSN axon coalescence and the formation of OB glomeruli.

\section{Functional characterization of tenascin effects on OSN neurite outgrowth}

As Tnc is in a position to influence OSN axon growth in the developing $\mathrm{OB}$, we developed an in vitro assay to assess the effects of this candidate inhibitory cue. Microexplants of OE were generated by a brief ( $5 \mathrm{~min}$ ) enzymatic digestion of OE harvested from postnatal mice followed by gentle trituration. This treatment results in small explants that can be adhered to substrates and neurite outgrowth assessed. Substrates comprise PDL-coated glass coverslips $(50 \mu \mathrm{g} / \mathrm{ml})$ that are incubated with extracellular matrix proteins for $1 \mathrm{~h}$ at $37^{\circ} \mathrm{C}$. Control experiments were per- 


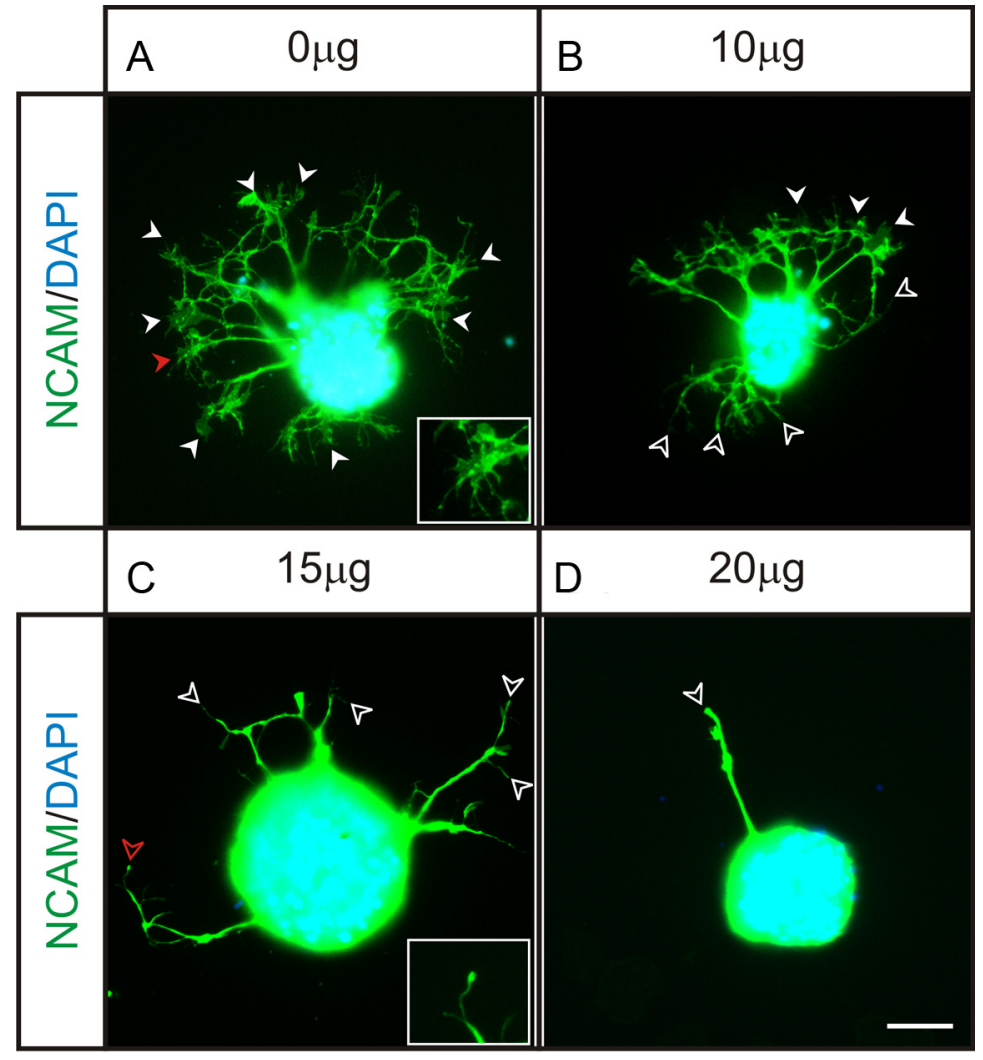

E OSN Neurite Outgrowth on Tnc substrate

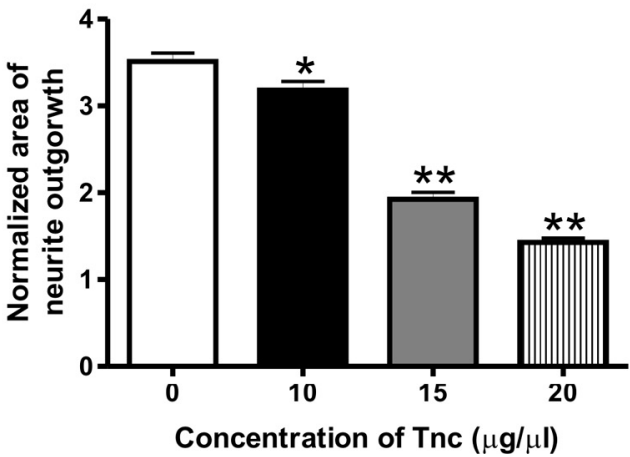

Figure 3. Dose-response of OSN neurite outgrowth on Tnc. Microexplants of olfactory epithelium plated on PDL-coated coverslips $(50 \mu \mathrm{g} / \mathrm{ml})$ that are subsequently coated with increasing concentrations of Tnc. Explants were grown for $48 \mathrm{~h}$, fixed, and stained with NCAM (green) and DAPI (blue), and neurite outgrowth was assessed. $\boldsymbol{A}$, In the absence of Tnc, OSNs extend neurites, and large spread growth cones are observed [arrowheads; see inset (high-magnification of growth cone indicated with red arrowhead)]. $\boldsymbol{B}$, Explants grown on $10 \mu \mathrm{g} / \mathrm{ml}$ Tnc appear to have shorter neurites and fewer spread growth cones (solid arrowheads), with more neurites ending in blunt bull-nosed type growth cones (open arrowheads). $\boldsymbol{C}$, The neurites extending from explants grown on $15 \mu \mathrm{g} / \mathrm{ml}$ Tnc were sparse, considerably less branched and tapered to blunt endings [open arrowheads; see inset (high-magnification of growth cone indicated with red open arrowhead); compare inset in $\boldsymbol{A}$ ]. No spread growth cones were observed. $\boldsymbol{D}$, Explants grown on $20 \mu \mathrm{g} / \mathrm{ml}$ Tnc were occasionally observed to extend neurites. Often no neurites were extended (see C. When neurites were extended, they had few or no branches and tapered to a blunt end (open arrowhead). E, Quantification of dose-response of neurite outgrowth on Tnc. Neurite outgrowth was determined by measuring total area of NCAM staining and dividing by the area of DAPI staining, to normalize for variability in explant size. Approximately 50 explants (49, 54, 47, 55, respectively) were analyzed for each condition (taken from 3 independent replicates) and analyzed using a one-way ANOVA with Dunnett's multiple-comparison post hoc test. Significant differences were observed in the ANOVA ( $p<0.0001)$, and tenascin-C was found to be an inhibitory molecule for OSN neurite outgrowth, with significant differences found between control $(0 \mu \mathrm{g} / \mathrm{ml}$ Tnc) and each Tnc concentration tested in a dose-dependent manner using Dunnett's multiple-comparison test: 0 versus 10, $p<$ $0.05 ; 0$ versus $15, p<0.01 ; 0$ versus $20, p<0.01$. Error bars indicate \pm SEM. ${ }^{*} p<0.05 .{ }^{* *} p<0.01$. Scale bar, $50 \mu \mathrm{m}$.

formed on PDL alone; on a known potent promoter of OSN neurite outgrowth, laminin; and on another tenascin family member, Tnr, to determine the specificity of the effect. When OSN explants were grown on PDL, modest neurite outgrowth was observed (Fig. 2A). When laminin $(20 \mu \mathrm{g} / \mathrm{ml})$ was added to the substrate, as expected from many studies in the literature (Kafitz and Greer, 1998), neurite outgrowth from OSN explants was greatly potentiated (Fig. 2B). However, when Tnc $(20 \mu \mathrm{g} / \mathrm{ml})$ was added to the substrate, almost no neurite outgrowth was observed (Fig. 2C), with axons preferring to extend within the explant itself rather than onto the substrate (green ring of axons extending around the edge of the explant) (Fig. 2C). In contrast, when Tnr $(20 \mu \mathrm{g} / \mathrm{ml})$ was provided, there was no effect on OSN neurite outgrowth (Fig. $2 D$ ), with explants appearing indistinguishable from those grown on PDL alone (compare Fig. 2A).

To characterize this inhibitory effect of Tnc on OSN neurite outgrowth, we determined the dose-response of Tnc as a substrate (Fig. 3). Approximately 50 explants were analyzed for each condition (taken from three replicates) and analyzed using a one-way ANOVA with Dunnett's multiple-comparison post hoc test. Tnc was found to be inhibitory for OSN neurite outgrowth, with significant differences observed via ANOVA $(p<0.0001)$. Even at the lowest concentration tested, $10 \mu \mathrm{g} / \mathrm{ml}$, a significant decrease in neurite outgrowth was observed $(p<0.05)$. At 15 $\mu \mathrm{g} / \mathrm{ml}$ Tnc, OSN neurites were sparse and considerably less branched (Fig. 3C), which was further reduced at $20 \mu \mathrm{g} / \mathrm{ml}$ when occasional, but often no, neurites were observed (Fig. 3D) (compare Fig. $2 C$ ). Effects were also observed on growth cones. On PDL alone, growth cones were spread with many filopodia (Fig. 3A, arrowheads; inset is a high-magnification image of the spread growth cone indicated with a red arrowhead). On 10 $\mu \mathrm{g} / \mathrm{ml}$ Tnc, explants had fewer spread growth cones (Fig. 3B, arrowheads), with more neurites ending in blunt "bull-nosed" type growth cones (Fig. $3 B$, open arrowheads). At $15 \mu \mathrm{g} / \mathrm{ml} \mathrm{Tnc,}$ no spread growth cones were observed; all processes tapered to blunt endings (Fig. $3 C$, open arrowheads; inset is a high magnification image of the bull-nose growth cone indicated with the red open arrowhead). Similarly, at $20 \mu \mathrm{g} / \mathrm{ml}$ Tnc, when neurites were present they tapered to a blunt end (Fig. 3D, open arrowhead).

Our dose-response assays were performed when Tnc was presented as a uniform substrate. However, Tnc is present in the $\mathrm{OB}$ in vivo in a boundary-like pattern. To determine the response of OSN neurite outgrowth to Tnc boundaries, we performed a modified stripe assay (Knöll et al., 2007), in which explants were plated on a substrate with interdigitating stripes of 


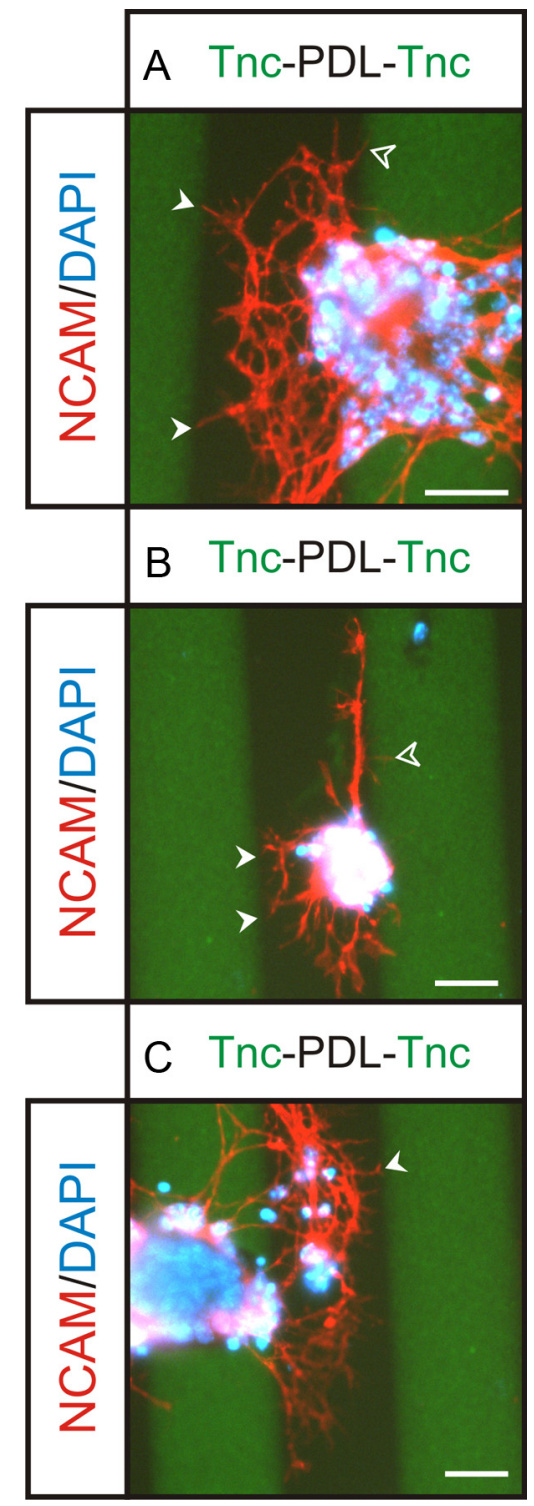

Figure 4. Modified stripe assay of neurite outgrowth on Tnc. To assess OSN neurite behavior at Tnc boundaries, $0 \mathrm{E}$ explants were plated on stripes of Tnc (green) interdigitating with PDL (black). Explants were grown for $48 \mathrm{~h}$, fixed, and stained with NCAM (red) and DAPI (blue). Substrate was stained for Tnc (green) so boundaries could be visualized. $\boldsymbol{A}$, Explants were observed to preferentially extend neurites on PDL stripes, with few being extending on the green Tnc stripes. Within the PDL stripes, neurites extensively branched, but stopped at Tnc boundaries (solid arrowheads). One process appears to have crossed the boundary onto the Tnc stripe (open arrowhead), but it is very thin and has not grown far. It is likely that this thin process is sampling the substrate and in the process of retracting. $\boldsymbol{B}$, An explant that was observed to extend neurites along the Tnc boundary. Note the many neurites that stopped at Tnc boundaries (solid arrowheads). Again some fine processes were seen to extend onto the Inc stripe (open arrowhead), which likely reflect sampling of the environment. The polarized growth from this explant preferentially in the PDL stripe versus the Tnc stripe indicates the preference of OSN axons to not grow on Tnc substrates. C, Another example of the preferential growth and branching on PDL versus Tnc. Note the neurite that has stopped at the Tnc boundary (arrowhead). Scale bars, $50 \mu \mathrm{m}$.

PDL and Tnc (Fig. 4). Neurites preferentially grew out of the explant on PDL stripes, with little (Fig. $4 A, C$ ) or no (Fig. $4 B$ ) outgrowth from the explants on Tnc stripes. Branching was extensive on PDL stripes, but neurites stopped at Tnc boundaries (Fig. $4 A$, arrowheads). Some neurites turned and grew along the boundary (Fig. 4B). Occasional fine processes entered a Tnc stripe (Fig. $4 A, B$, open arrowheads), but these were rare in- stances and extension onto the Tnc stripe was minimal after $48 \mathrm{~h}$ in vitro. Furthermore, it is of note that the fine processes that did extend slightly onto the Tnc stripe were secondary neurite branches and not primary neurites originating from within the explant.

To confirm that the purified chick Tnc was not contaminated with other inhibitory proteins like chondroitin sulfate proteoglycans (CSPGs), which can copurify with Tnc and might confound interpretation, we performed two control experiments (supplemental Fig. 2, available at www.jneurosci.org as supplemental material). First, we did not detect any extra bands using a silver stain (supplemental Fig. $2 \mathrm{~A}$, available at www.jneurosci.org as supplemental material), and we repeated the neurite outgrowth assay, after treating the TNC substrate with chondroitinase ABC, and did not see any change in response (supplemental Fig. $2 B$, available at www.jneurosci.org as supplemental material). Therefore, we do not believe the Tnc protein is significantly contaminated with other inhibitory proteins and the effect observed is attributable to Tnc.

These data demonstrate that Tnc is an important boundary molecule influencing OSN axon outgrowth. Coupled with its expression pattern in vivo, this provides compelling evidence that Tnc may be an important determinant in the establishment of the primary olfactory pathway from the OE to the OB. Of additional interest, primary neurites extending from explants robustly avoided Tnc boundaries, but there was some evidence that secondary branches may be less subject to the inhibitory effects of Tnc.

\section{Olfactory bulb development in Tnc-null mice}

Given that we have demonstrated that Tnc is inhibitory to OSN neurite outgrowth in vitro, we next examined $\mathrm{OB}$ development in the absence of Tnc. We obtained tissue of Tnc ${ }^{-1-}$ mice and wildtype littermates $\left(\mathrm{Tnc}^{+/+}\right.$) during the period of glomerulogenesis, specifically, at E17, PND0, PND2, and PND4. To determine whether the absence of Tnc, as a putative inhibitory/boundary molecule, would lead to deficits in OB development, we examined two aspects of pathway formation: (1) synapse formation and (2) topography between $\mathrm{OE}$ and $\mathrm{OB}$.

\section{Synapse formation}

VGlut2 is restricted to the presynaptic compartment of axodendritic synapses made by OSN axons at their targets within glomeruli (Nakamura et al., 2005). As such, it can be used as a measure of OSN synaptogenesis in the developing OB. When comparing $\mathrm{Tnc}^{-/-}$mice and Tnc ${ }^{+/+}$littermates at E17 to PND4 stained with NCAM (red) and VGlut2 (green) (Fig. 5), some subtle differences were observed. Analysis was focused on the medial surface of the $\mathrm{OB}$ as previous studies have indicated this region shows more advanced development (Treloar et al., 1999). At E17 in wild-type mice, synapses (green) appeared in a thin layer at the interface of the olfactory nerve ONL and deeper layers of the OB. Protoglomeruli (i.e., immature glomeruli) were observed as hemicircular thickenings at the site of VGlut2 staining (Fig. 5A, arrowheads). They remained fused with the ONL, a feature characteristic of protoglomeruli. At E17 in null mice, synapses (green) were less dense compared with wild types (Fig. 5B) (compare Fig. $5 A$ ), with the layer of VGlut2 staining at the edge of the ONL appearing very thin and irregularly stained. Protoglomeruli were rarely, if ever, observed. Although some regions were identified with increased density of VGlut2 staining (Fig. 5B, open arrowheads), they were not hemicircular, did not protrude 


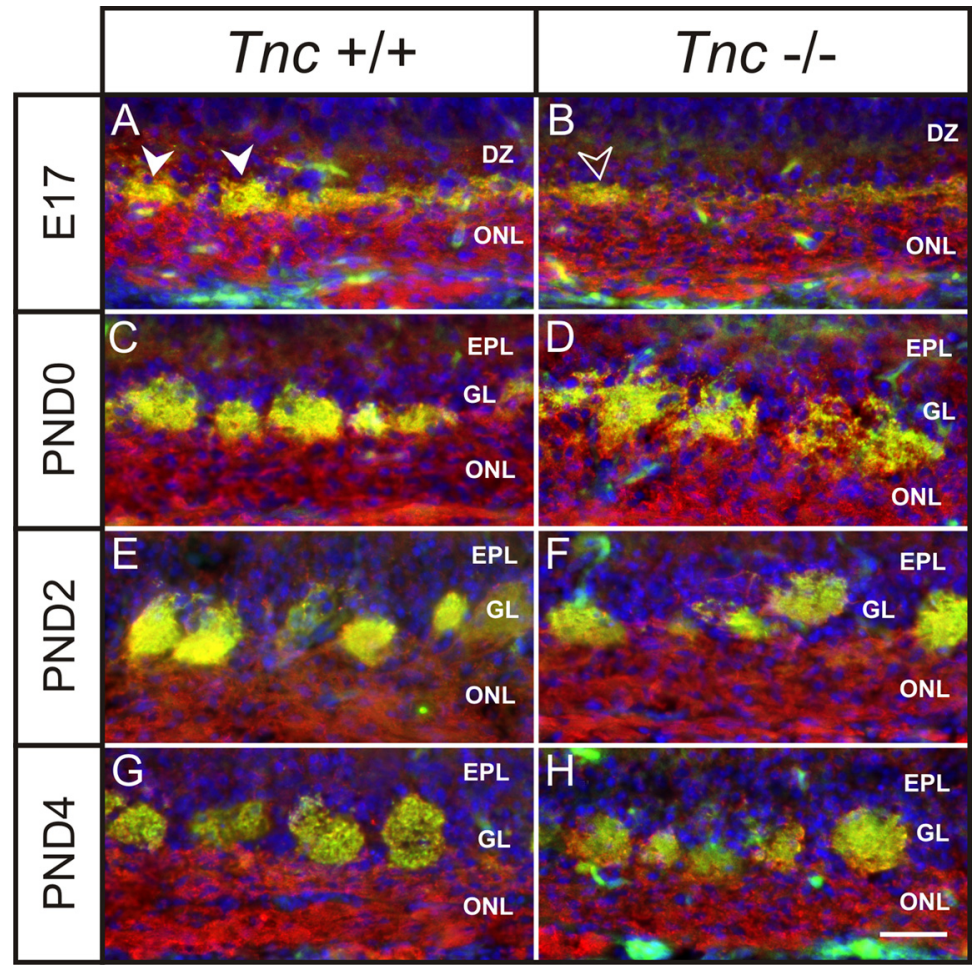

Figure 5. Glomerular development in $\mathrm{Tnc}^{-/-}$mice. $\boldsymbol{A}-\boldsymbol{H}$, Coronal sections of $\mathrm{Tnc}^{+/+}$and $\mathrm{TnC}^{-/-}$medial olfactory bulb stained for the synaptic marker VGlut2 (green), the marker of OSN axons Ncam (red), and the nuclear marker DAPI (blue) at E17 ( $A$, $\boldsymbol{B}), \operatorname{PNDO}(\boldsymbol{C}, \boldsymbol{D}), \operatorname{PND2}(\boldsymbol{E}, \boldsymbol{F})$, and PND4 $(\boldsymbol{G}, \boldsymbol{H}) . \boldsymbol{A}$, In wild types at E17, VGlut2 staining is observed to be in a layer at the interface of the ONL and the DZ. Hemicircular synaptic thickenings were observed in this layer (arrowheads), which are the immature glomeruli, or protoglomeruli, that are still fused and not separated from the ONL. $\boldsymbol{B}$, In null mice at E17, VGlut2 staining is also observed to be in a layer at the interface of the $0 \mathrm{NL}$ and $\mathrm{DZ}$, but considerably less dense than controls (compare $A$ ). Protoglomeruli were rarely, if ever, observed. Some thickening in the layer of VGlut2 staining were occasionally observed (open arrowheads); however, they were not hemicircular and did not protrude into the DZ (compare $\boldsymbol{A}$, arrowheads). $\boldsymbol{C}$, In wild types at PND0, glomeruli are discrete neuropil, separated from the $0 \mathrm{NL}$ and forming their own layer, the glomerular layer (GL). EPL, External plexiform layer. $D$, In null mice at PNDO, glomeruli do not appear like the discrete structures seen in wild types. Rather, they have a more diffuse, shaggy appearance and are not separated from the underlying nerve layer. These structures also appear larger than control glomeruli. $\boldsymbol{E}$, In wild types at PND2, glomeruli appear to contain a high density of synapses. $\boldsymbol{F}$, In null mice at PND2, glomeruli appear more developed compared with PND0. They appear more densely innervated (less shaggy) and separated from the ONL into a distinct GL. $\boldsymbol{G}$, In wild types at PND4, glomeruli appear to have increased in size compared with PND2 (compare $\boldsymbol{E}$ ). $\boldsymbol{H}$, In null mice at PND4, glomeruli appear indistinguishable from controls. Scale bar, $100 \mu \mathrm{m}$.

into the dendritic zone, and were rarely observed (Fig. 5, compare $B$, open arrowheads; $A$, arrowheads).

By PND0, glomeruli were distinct discrete neuropil in wildtype animals (Fig. 5C), but in Tnc-null mice, glomeruli presented a more "shaggy" appearance (Fig. 5D), suggesting that the OSN axons had not fully coalesced into these structures. Similarly, glomerular-like structures in the null mice were not discrete or separated from the nerve layer, hence appearing immaturemore like the protoglomeruli in wild-type mice at E17 (Fig. 5, compare $D, A$ ). By PND2, wild-type glomeruli appeared to have further coalesced and to contain a high density of synapses (Fig. $5 E$ ). In contrast, null glomeruli at PND2 did not appear as tightly coalesced and continue to express an irregular outline (Fig. 5F), appearing more like PND0 wild-type glomeruli (compare Fig. 5C). At PND4, wild-type (Fig. 5G) and null (Fig. 5H) glomeruli were indistinguishable.

To determine whether differences existed across the rostrocaudal axis of the OB, we examined sections every $100 \mu \mathrm{m}$ along the rostrocaudal extent of the $\mathrm{OB}$ (data not shown). To quantify the differences we observed, we measured the diameter of glomeruli in null and wild-type mice from PND0 to PND4. Glomeruli were defined using three measures: (1) VGlut2 staining, (2)
NCAM staining, and (3) a nuclear stain (Draq5). Collectively, these defined the boundaries of developing glomeruli in these mice. Glomerular diameter was measured in the plane parallel to the ONL on the medial surface of $\mathrm{OB}$ using MetaMorph software. Statistical differences were determined using an unpaired Student $t$ test (Fig. 6A). We could not perform this analysis at E17 since even protoglomeruli were not yet readily distinguished from the ONL and because VGlut2 staining appeared as a continuous layer without evidence of the hemicircles indicative of protoglomeruli.

At PND0, glomeruli had a significantly larger diameter $[50.75 \pm 1.46 \mu \mathrm{m}(n=$ 217 ) in null mice compared with wildtype controls, $37.47 \pm 1.23 \mu \mathrm{m}(n=$ 253)]. At PND2, glomeruli have decreased (or coalesced) to an average diameter of $41.28 \pm 1.05 \mu \mathrm{m}(n=303)$ in the Tncnull mice, but this is still significantly larger compared with wild-type glomeruli with an average diameter of $38.03 \pm 0.95$ $\mu \mathrm{m}(n=343)$. However, by PND4, both wild-type and null glomeruli had increased in size from PND2 but were no longer significantly different from each other [null, $43.62 \pm 1.35 \mu \mathrm{m}(n=212)$; wild type, $42.44 \pm 0.99 \mu \mathrm{m}(n=298)]$.

This analysis averaged glomerular diameter across the whole bulb and therefore may have missed subtle changes within the population of glomeruli. Therefore, to more fully characterize the phenotype, we examined the frequency distributions (histograms) of glomerular diameter. At PND0 (Fig. 6B), the Tncnull histogram is skewed to the left of the wild-type curve, with the peak $(23 \%$ of glomeruli) around $50 \mu \mathrm{m}$ compared with the wild type with a peak (27.7\% of glomeruli) at $30 \mu \mathrm{m}$ ( $54.6 \%$ occur in bins 20 and $30 \mu \mathrm{m})$. By PND2, the null histogram has moved toward the right, closer to the wild-type curve (Fig. $6 \mathrm{C})$. The peak $(22.4 \%$ of glomeruli) of the null is around $40 \mu \mathrm{m}$, whereas the peak of the wild type is around $30 \mu \mathrm{m}$ (23.3\% of glomeruli). By PND4, both null and wild type have peaks (27.8 and $24.2 \%$, respectively) that occur around $40 \mu \mathrm{m}$ (Fig. $6 D$ ). These data demonstrate that glomeruli have a larger diameter in younger Tnc-null mice, but as development continues, coalescence of axons continues until glomeruli are indistinguishable from wild-type controls.

\section{Topography}

As axon coalescence is initially impaired in these animals, we also investigated whether appropriate topography between epithelium $(\mathrm{OE})$ and bulb was maintained. In preliminary studies, we used antibodies against the odor receptor MOR28 (Barnea et al., 2004). However, although these antibodies did identify MOR28expressing OSNs in the OE, they only did so using peroxidase detection, not immunofluorescence, and staining for MOR28 was not observed in OSN axons via either method (data not 
A Average Glomerular Diameter

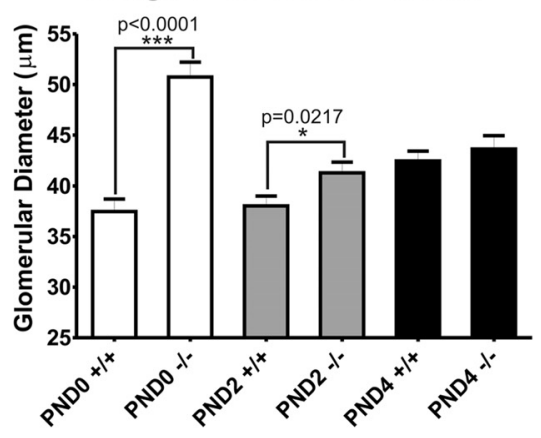

C

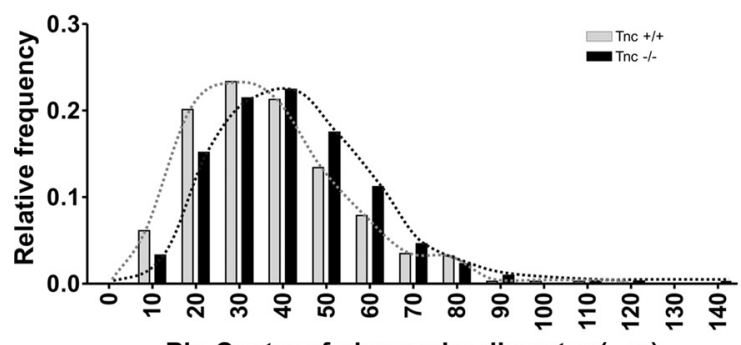

Bin Center of glomerular diameter $(\mu \mathrm{m})$
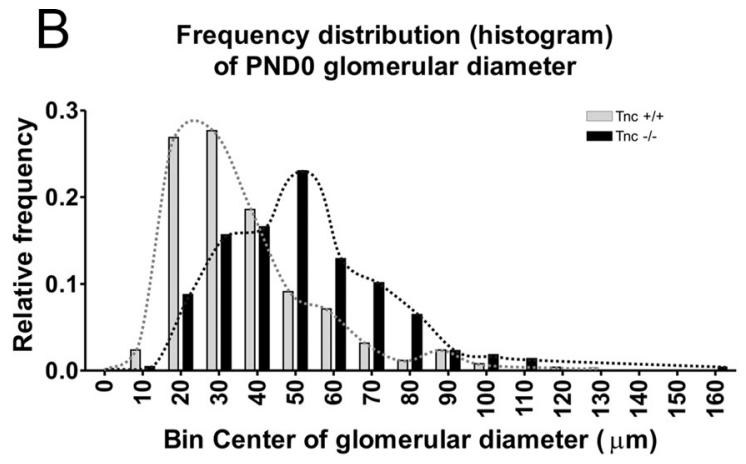

Frequency distribution (histogram) of PND4 glomerular diameter

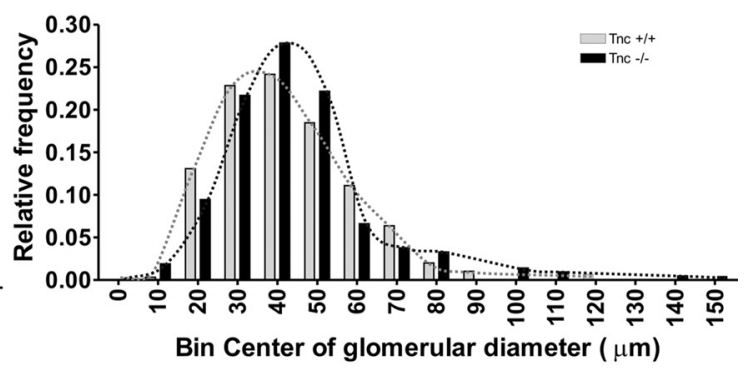

Figure 6. Quantification of glomerular development in $\mathrm{TnC}^{-/-}$mice. $A$, Glomerular diameter was measured (parallel to the ONL) using MetaMorph software on the medial surface of OB of $\mathrm{Tnc}^{+/+}$and $\mathrm{Tnc}^{-/-}$mice at PNDO, PND2, and PND4. Glomeruli were identified as being both NCAM (red) and VGlut2 (green) positive and glomerular boundaries confirmed with DAPI staining (Fig. 5). At PND0, glomeruli were observed to have a significantly larger diameter ( $50.75 \pm 1.46 \mu \mathrm{m} ; n=217)$ in null mice compared with wild-type controls (37.47 $\pm 1.23 \mu \mathrm{m} ; n=253)$ with $p<$ 0.0001 using an unpaired Student's $t$ test. At PND2, glomeruli have coalesced in the null mice to $41.28 \pm 1.05 \mu \mathrm{m}(n=303)$, which is still significantly larger compared with wild-type glomeruli,

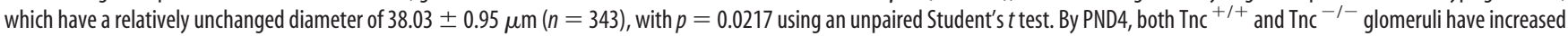
in size from PND2 and are not significantly different from each other [value of $p=0.4709$; wild type, $42.44 \pm 0.99 \mu \mathrm{m}(n=298) ;$ null, $43.62 \pm 1.35 \mu \mathrm{m}(n=212)$ ]. Error bars indicate \pm SEM. ${ }^{*} P<0.05$, ${ }^{* * *} P<0.0001$. B , Histogram of glomerular diameter at PNDO. In Tnc ${ }^{+/+}$mice, the peak of the curve was located between bins 20 and $30 \mu \mathrm{m}$, with $54.6 \%$ of glomeruli residing in these bins. In contrast, in $\mathrm{Tnc}^{-1-}$ mice, the peak was located at $50 \mu \mathrm{m}$, with $23 \%$ glomeruli residing in this bin. Thus, the frequency distribution was clearly shifted to the left, indicating larger glomerular diameter size. C, At PND2, the $\mathrm{Tnc}^{-1-}$ frequency distribution curve had shifted more to the right, compared with PNDO, and was much closer to the $\mathrm{Tnc}^{+/+}$distribution curve. The peak of the $\mathrm{Tnc}^{-1-}$ curve occurs at $40 \mu \mathrm{m}$ (bin contains $22.4 \%$ of glomeruli), whereas the peak of the $\mathrm{Tnc}^{+/+}$curve is around $30 \mu \mathrm{m}$ (bin contains $23.3 \%$ of glomeruli). D, By PND4, both Tnc ${ }^{-/-}$and $\mathrm{Tnc}^{+/+}$curves have peaks that occur around $40 \mu \mathrm{m}$ (bins contain 27.8 and $24.2 \%$ glomeruli, respectively).

shown). Therefore, we turned to an alternate marker of a subset of OSN axons that project topographically to the OB. The plant lectin DBA labels a subset of OSN axons that project predominantly to the dorsomedial OB (Key and Akeson, 1993) and has previously proved useful to assess changes in topographic projections (Treloar et al., 1996a, 1997). We performed this analysis at multiple ages, but present here only PND0 (Fig. 7), the age with the most pronounced deficit in glomerular development (see above). In wild-type mice, DBA (Fig. $7 A$, red) bound to OSN axons that target predominantly the dorsal and medial $\mathrm{OB}$. These sections were matched in the rostrocaudal axis for both wild-type and Tnc-null mice as closely as possible, using anatomical features of the nasal cavity and surrounding tissue landmarks as guides. In null mice, axons bound by DBA (Fig. 7B, red) targeted the same regions of the $\mathrm{OB}$ when compared with wild-type controls (compare Fig. 7A). Although fine targeting deficits may not be revealed using this approach, it should be noted that wild-type mice axons expressing the same odor receptor show small variations in their relative position (Strotmann et al., 2000). Thus, we conclude that at least the gross topographic projection of OSN axons from the OE to the OB appears mostly intact in Tnc-null mice.

\section{Phosphacan}

Tnc interacts with many components of the ECM. Complex and frequently overlapping patterns of expression have been reported with phosphacan, a CSPG (Garwood et al., 2001). To determine whether these interactions were also occurring in the developing $\mathrm{OB}$, we performed double-label immunofluorescence localization studies using antibodies against Tnc and phosphacan (Fig. 8). As is evident by colocalization (yellow), Tnc and phosphacan expression patterns partially overlap in the developing $\mathrm{OB}$ at all ages examined. This finding was somewhat surprising in light of our report that 6-sulfated CSPGs are not detected in the developing OB until E17 (Shay et al., 2008). We therefore conclude that phosphacan does not carry 6-CS side chains until at least E17, when sulfation may change. Regardless, these data demonstrate that phosphacan, like Tnc, is also present in a boundary-like pattern early in olfactory pathway development and may influence OSN axons. However, it is interesting to note that phosphacan is found more extensively within glomerular structures than TNC (arrowheads at PND2 and PND4). It must be noted, however, that phosphacan is a splice variant of Ptprz (protein tyrosine phosphatase receptor type $\zeta$ ), so staining may reflect receptor and/or proteoglycan isoforms. This remains to be determined.

\section{Discussion}

Here, we show Tnc is an inhibitory boundary molecule developmentally regulated in the olfactory system. Our data support the hypothesis that Tnc within the $\mathrm{OB}$ is a regulatory mechanism governing both spatial and temporal parameters of OSN outgrowth and glomerulogenesis. Furthermore, we show that the 


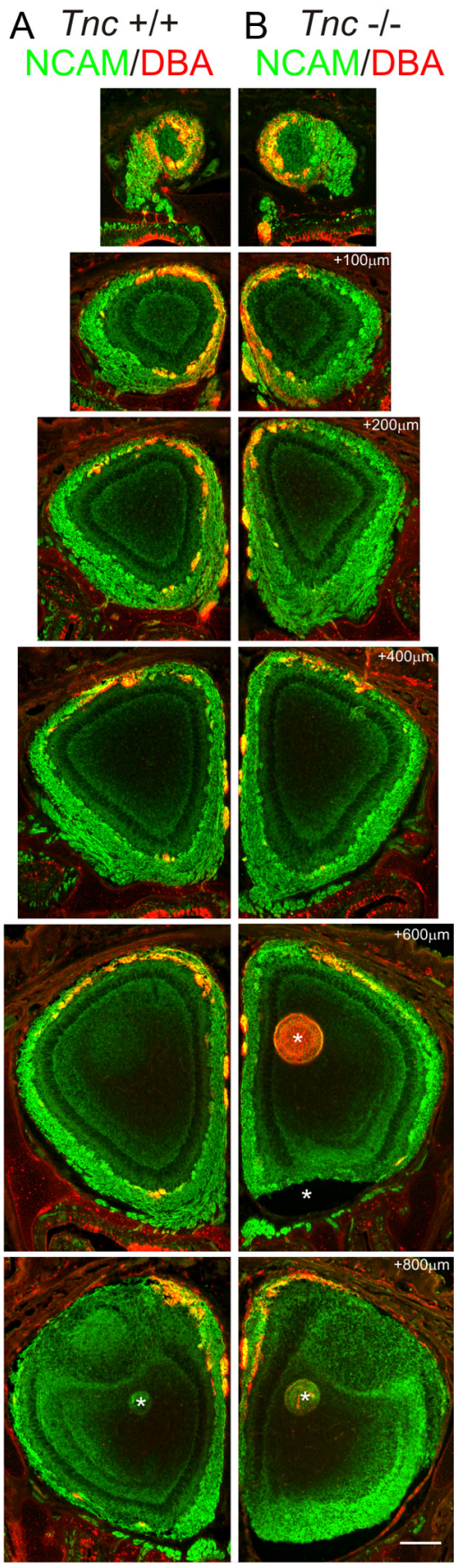

Figure 7. Topography in neonatal $\mathrm{TnC}^{-/-}$mice. $\boldsymbol{A}, \boldsymbol{B}$, Coronal serial sections taken every $100 \mu \mathrm{m}$ from rostral to caudal of olfactory bulb of PNDO $\mathrm{TnC}^{+/+}(\boldsymbol{A})$ and $\mathrm{Tnc}^{-1-}(\boldsymbol{B})$ mice, stained for Ncam (green) and with the plant lectin DBA (red). DBA binds a subset of OSN axons that project predominantly to the dorsomedial OB (Key and Akeson, 1993). As expected, in $\mathrm{TnC}^{+/+}$mice, DBA+ OSN axons were found predominantly in the dorsal and medial OB. In null mice, $\mathrm{DBA}+$ axons were also observed to target the dorsomedial OB. No obvious differences were observed in the topography of this subset of OSN axons. Scale bar, $250 \mu \mathrm{m}$. *Artifact from tissue sectioning.

role of Tnc is ubiquitous across all OSNs and does not differentially affect the $\mathrm{OE}$ to $\mathrm{OB}$ topography. To our knowledge, this is the first identification of a molecular "stopwatch" regulating $\mathrm{OB}$ glomerulogenesis.

OSN axons emerge from the OE, coalesce into fascicles, and approach the presumptive $\mathrm{OB}$ before achieving molecular heterogeneity. As they form the ONL, the axons reorganize, coalesce into molecularly defined fascicles from cells expressing the same
$\mathrm{OR}$, and penetrate the dendritic zone of the $\mathrm{OB}$ in which they initially appear as protoglomeruli (Treloar et al., 1996b, 1999). Within protoglomeruli, the OSN axons establish excitatory synapses with projection neurons, mitral/tufted cells, and subpopulations of interneurons, periglomerular cells. By birth, glomeruli are discrete structures, separated from the ONL (Treloar et al., 1999). Although glomerular development continues postnatally (Kim and Greer, 2000) during the early perinatal period, responses to odors are easily demonstrated behaviorally (de Chevigny et al., 2006) and physiologically (Greer et al., 1982).

Our current data are consistent with this model and show that protoglomeruli are apparent in wild-type mice at E17. As we previously described, protoglomeruli, although not yet distinct from the ONL, appear as hemicircles protruding from the ONL and into the underlying dendritic zone (Treloar et al., 1999). In contrast, in the absence of Tnc, although there is evidence of regions of increased density of VGlut2 puncta at the interface of the ONL, there are no protoglomeruli until PND0. Even as late as PND2, we found significant differences between the diameter of glomeruli in $\mathrm{Tnc}^{-1-}$ and $\mathrm{Tnc}^{+/+}$mice. Given our in vitro assays showing the boundary effect of Tnc on OSN axon outgrowth, our data are consistent with the hypothesis that Tnc plays a pivotal role in glomerulogenesis, in both initiation and refinement.

When initially generated, $\mathrm{Tnc}^{-1-}$ mice were reported to have no phenotype (Saga et al., 1992). More recently, Tnc-deficient mice generated by Evers et al. (2002) showed many subtle phenotypes. For example, in the hippocampus, Tnc has been implicated in synaptic plasticity. Tnc ${ }^{-1-}$ mice also have impaired low-frequency induced long-term potentiation (Evers et al., 2002) and deficits in hippocampus-dependent contextual memory (Strekalova et al., 2002). Tnc here may be acting, at least in part, through regulation of L-type voltage-dependent calcium channels or sodium channels, both of which can bind Tnc (Srinivasan et al., 1998) (for review, see Dityatev and Schachner, 2006).

Similar to the boundary-like pattern in the OB, Tnc defines the boundaries of barrels in mouse somatosensory cortex during the first postnatal week (Steindler et al., 1989). CybulskaKlosowicz et al. (2004) used 2-deoxyglucose activity mapping to examine barrel fields in $\mathrm{Tnc}^{-1-}$ mice. Similar to the decreased synaptic plasticity in the adult $\mathrm{Tnc}^{-1-}$ hippocampus, the plasticity was also diminished in the cortical barrel fields with significantly less lesion-induced expansion of vibrissae cortical representation compared with controls. Decreased functional plasticity in somatosensory cortex after vibrissectomy highlights the point that the barrel cortex appears normal in adult $\mathrm{Tnc}^{-1-}$ mice but is less effective in remodeling of synaptic connectivity than controls. In light of our developmental data showing delays in glomerulogenesis, it would be interesting to see whether similar delays occur in barrel formation in $\mathrm{Tnc}^{-1-}$ mice. Likewise, it would be equally interesting to see whether reduced functional plasticity (i.e., odor induced learning) occurs in adult $\mathrm{Tnc}^{-/-}$mice.

$\mathrm{Tnc}^{-1-}$ mice do display delayed onset of odor detection (de Chevigny et al., 2006), a discrete phenotype not unlike that described above for the hippocampus. However, the mechanisms underlying delays in the onset of odor detection were not clear. Our data now demonstrate that the delay in the acquisition of this odor-directed behavioral task most likely reflects the parallel delay in the formation of OB glomeruli. The slight temporal shift seen in their odor detection data is consistent with an additional retardation in the formation of the glomerular synaptic circuits, including primary afferent as well as local circuit connections (Kim and Greer, 2000). Similar to our data, they also show that 
the delayed acquisition of the odor preference is transient and is corrected as development continues.

What is the mechanism by which Tnc regulates glomerulogenesis? The cellular distribution of Tnc likely contributes to a mechanism that would underlie the earliest steps of glomerular formation, when axons begin exiting the ONL and coalescing within the dendritic zone. As discussed in Introduction, myriad mechanisms are implicated in the coalescence of axons and glomerulogenesis. The data presented here are consistent with a model in which Tnc contributes to a combinatorial code governing these events, as proposed by Key and St John (2002). We suggest that the Tnc-associated delay reflects an imbalance of cues affecting OSN axons. The later resolution of glomerular targeting/structure indicates the loss of a single cue is insufficient to prevent the complete formation of this sensory pathway. This is consistent with perturbations in functional activity (CNG2A, ACIII, $\mathrm{G}_{\mathrm{olf}}$ ), guidance molecules (Kirrel2, Kirrel3, Sema 3A, Npn-1, Plexin-A1, OCAM, ephrin-A5/EphA5, Big2), and cell surface carbohydrates ( $\beta 3 \mathrm{GnT} 1)$ (for review, see Imai and Sakano, 2007, 2008).

We cannot discount the possibility that the effects observed in the $\mathrm{Tnc}^{-1-}$ are secondary to postsynaptic mechanisms. For example, Tnc is expressed developmentally by radial glia (Bartsch et al., 1992; Stoykova et al., 1997; Götz et al., 1998), which could affect the migratory behavior of $\mathrm{OB}$ cells. Moreover, Tnc is expressed in the ventricular and subventricular zones and is a regulator of embryonic neural stem cell behavior, but apparently not that of adult stem cells (Garcion et al., 2004; Kazanis et al., 2007). Moreover, it does not appear that migration of neuroblasts into the OB from the rostral migratory stream is altered in adult Tnc-null mice (de Chevigny et al., 2006). Consequently, given our in vitro data showing Tnc acting as a strong boundary molecule for OSN axons, we believe the most plausible mechanism is a primary effect on the OSN axons. During development, a waiting period for axons targeting specific regions occurs throughout the brain and is thought to be important for the formation of proper neural networks (Schreyer and Jones, 1982; Ghosh and Shatz, 1992; Ozaki and Snider, 1997; Wang and Scott, 2000). For example, the projection pattern of dorsal root ganglion axons is delayed between the formation of the dorsal funiculus and the extension of collaterals into the dorsal mantle layer (Ozaki and Snider, 1997). OSN axons have a similar waiting period when they first grow into the $O B$ and form an ONL, before extending into the dendritic zone (DZ) and initiat-

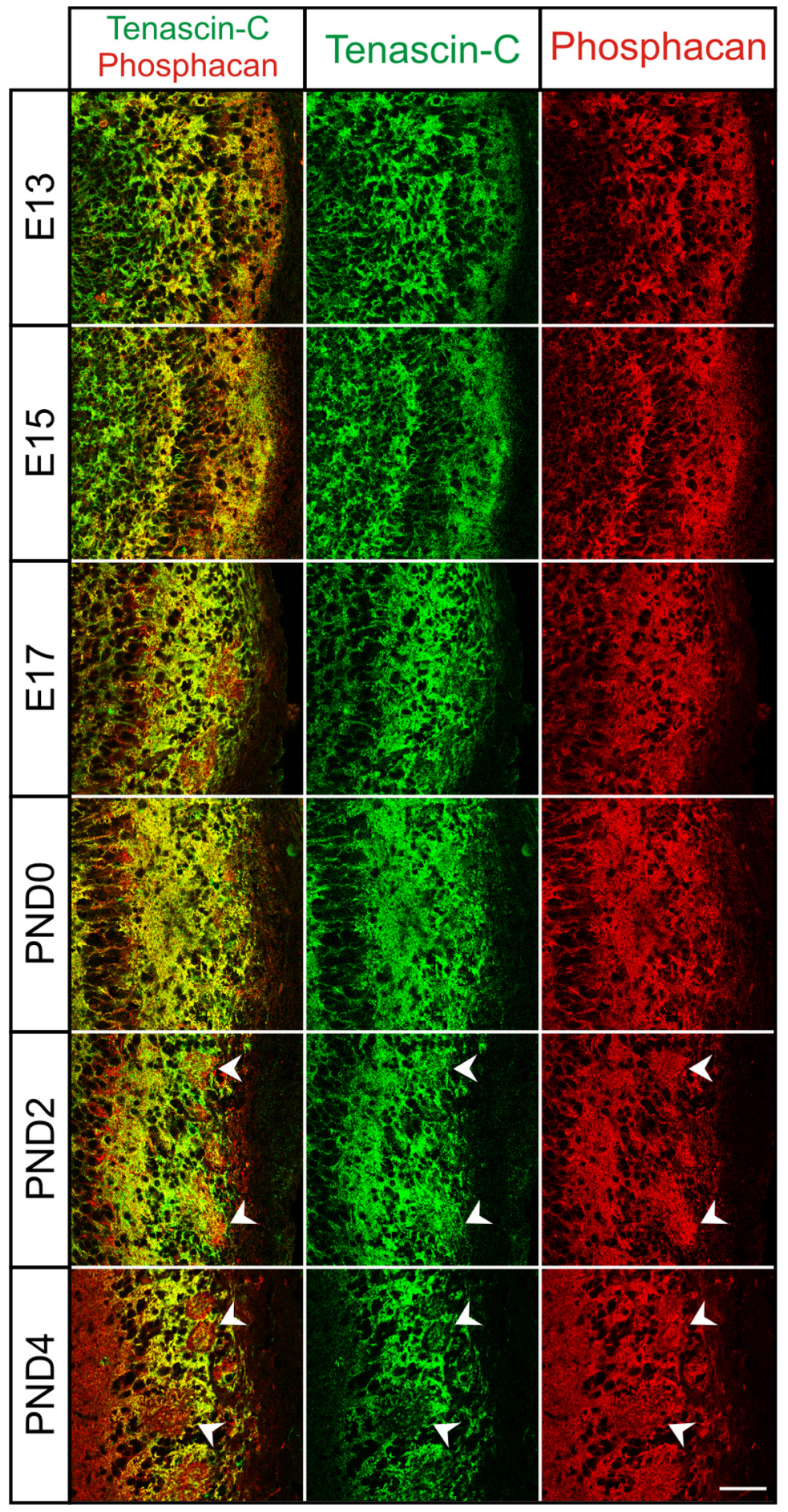

Figure 8. Colocalization of Tnc and Phosphacan in the developing OB. Coronal section of OB from E13-E17 and PND0 -PND4 stained with Tnc (green) and phosphacan (red). Tnc and phosphacan partially colocalize, as evident by yellow overlap. However, distinct red and green staining is also evident. Phosphacan is found more extensively within glomerular structures than TNC (arrowheads). Both proteins are in a position to influence OSN axon growth. Scale bar, $50 \mu \mathrm{m}$.

ing glomerulogenesis (Treloar et al., 1999; Renzi et al., 2000). Inhibitory guidance cues have been identified, which transiently prevent sensory afferents from penetrating the dorsal spinal cord during the waiting period (Watanabe et al., 2006). In the chick OB, class-3 semaphorins were identified as repellants that prevent OSN axons from prematurely entering the OB (Renzi et al., 2000). Here, we demonstrate delayed glomerular development in mice lacking Tnc, providing the first evidence in the mammalian 
olfactory system of an inhibitory guidance cue necessary for glomerular formation, and therefore the formation of the primary afferent synapses.

Interestingly, we did not observe the premature entry of OSN axons into the deeper layers of the OB in the absence of Tnc, as we expected. In chick, OSN axons overshoot the OB and enter cortex in the absence of Sema3A (Renzi et al., 2000), and thus, we hypothesized a similar phenotype would be seen in the absence of Tnc. However, there are reports of phosphacan, a chondroitin sulfate proteoglycan, present in the developing mouse $\mathrm{OB}$ in a boundary pattern similar to Tnc (Clarris et al., 2000). We show that Tnc and phosphacan are present in mostly overlapping spatiotemporal patterns in the developing OB. Tnc and phosphacan also share multiple molecular interaction sites, with each other and with many of the same cell surface receptors, including cell adhesion molecules, other matrix proteins, and growth factors (for review, see Garwood et al., 2001). Our data suggest that other inhibitory cues like phosphacan, or other proteoglycans, are candidates to act in combination with Tnc in the $\mathrm{OB}$ to regulate the ingrowth of OSN axons. Regardless of the full nature of this combinatorial set of cues, our data clearly show that other molecules cannot fully compensate for the loss of Tnc (cf. Key and St John, 2002). Thus, we contend, in addition to the milieu of cues that allow OSN axons to sort and coalesce into functional glomeruli, another class of cues regulates the timing of $\mathrm{OB}$ development. Within the olfactory system, these global cues, which affect all OSNs, are necessary to coordinate developmental events like glomerulogenesis.

In summary, we demonstrate that Tnc regulates OSN axon growth, likely by inhibiting growth into the OB before the period of glomerular formation. In the absence of this molecule, glomerulogenesis is delayed, resulting in delayed onset of odor responsiveness.

\section{References}

Barnea G, O’Donnell S, Mancia F, Sun X, Nemes A, Mendelsohn M, Axel R (2004) Odorant receptors on axon termini in the brain. Science 304:1468.

Bartsch S, Bartsch U, Dörries U, Faissner A, Weller A, Ekblom P, Schachner M (1992) Expression of tenascin in the developing and adult cerebellar cortex. J Neurosci 12:736-749.

Bartsch S, Husmann K, Schachner M, Bartsch U (1995) The extracellular matrix molecule tenascin: expression in the developing chick retinotectal system and substrate properties for retinal ganglion cell neurites in vitro. Eur J Neurosci 7:907-916.

Chesler AT, Zou DJ, Le Pichon CE, Peterlin ZA, Matthews GA, Pei X, Miller MC, Firestein S (2007) A G protein/cAMP signal cascade is required for axonal convergence into olfactory glomeruli. Proc Natl Acad Sci U S A 104:1039-1044.

Clarris HJ, Rauch U, Key B (2000) Dynamic spatiotemporal expression patterns of neurocan and phosphacan indicate diverse roles in the developing and adult mouse olfactory system. J Comp Neurol 423:99-111.

Cutforth T, Moring L, Mendelsohn M, Nemes A, Shah NM, Kim MM, Frisén J, Axel R (2003) Axonal ephrin-As and odorant receptors: coordinate determination of the olfactory sensory map. Cell 114:311-322.

Cybulska-Klosowicz A, Zakrzewska R, Pyza E, Kossut M, Schachner M (2004) Reduced plasticity of cortical whisker representation in adult tenascin-Cdeficient mice after vibrissectomy. Eur J Neurosci 20:1538-1544.

de Chevigny A, Lemasson M, Saghatelyan A, Sibbe M, Schachner M, Lledo PM (2006) Delayed onset of odor detection in neonatal mice lacking tenascin-C. Mol Cell Neurosci 32:174-186.

Dityatev A, Schachner M (2006) The extracellular matrix and synapses. Cell Tissue Res 326:647-654.

Evers MR, Salmen B, Bukalo O, Rollenhagen A, Bösl MR, Morellini F, Bartsch U, Dityatev A, Schachner M (2002) Impairment of L-type $\mathrm{Ca}^{2+}$ channel-dependent forms of hippocampal synaptic plasticity in mice de- ficient in the extracellular matrix glycoprotein tenascin-C. J Neurosci 22:7177-7194.

Garcion E, Halilagic A, Faissner A, ffrench-Constant C (2004) Generation of an environmental niche for neural stem cell development by the extracellular matrix molecule tenascin C. Development 131:3423-3432.

Garwood J, Rigato F, Heck N, Faissner A (2001) Tenascin glycoproteins and the complementary ligand DSD-1-PG/phosphacan-structuring the neural extracellular matrix during development and repair. Restor Neurol Neurosci 19:51-64.

Ghosh A, Shatz CJ (1992) Pathfinding and target selection by developing geniculocortical axons. J Neurosci 12:39-55.

Götz M, Stoykova A, Gruss P (1998) Pax6 controls radial glia differentiation in the cerebral cortex. Neuron 21:1031-1044.

Greer CA, Stewart WB, Teicher MH, Shepherd GM (1982) Functional development of the olfactory bulb and a unique glomerular complex in the neonatal rat. J Neurosci 2:1744-1759.

Imai T, Sakano H (2007) Roles of odorant receptors in projecting axons in the mouse olfactory system. Curr Opin Neurobiol 17:507-515.

Imai T, Sakano H (2008) Odorant receptor-mediated signaling in the mouse. Curr Opin Neurobiol 18:251-260.

Imai T, Suzuki M, Sakano H (2006) Odorant receptor-derived cAMP signals direct axonal targeting. Science 314:657-661.

Joester A, Faissner A (1999) Evidence for combinatorial variability of tenascin-C isoforms and developmental regulation in the mouse central nervous system. J Biol Chem 274:17144-17151.

Kafitz KW, Greer CA (1998) The influence of ensheathing cells on olfactory receptor cell neurite outgrowth in vitro. Ann N Y Acad Sci 855:266-269.

Kaneko-Goto T, Yoshihara S, Miyazaki H, Yoshihara Y (2008) BIG-2 mediates olfactory axon convergence to target glomeruli. Neuron 57:834-846.

Kazanis I, Belhadi A, Faissner A, Ffrench-Constant C (2007) The adult mouse subependymal zone regenerates efficiently in the absence of tenascin-C. J Neurosci 27:13991-13996.

Key B, Akeson RA (1993) Distinct subsets of sensory olfactory neurons in mouse: possible role in the formation of the mosaic olfactory projection. J Comp Neurol 335:355-368.

Key B, St John J (2002) Axon navigation in the mammalian primary olfactory pathway: where to next? Chem Senses 27:245-260.

Kim H, Greer CA (2000) The emergence of compartmental organization in olfactory bulb glomeruli during postnatal development. J Comp Neurol 422:297-311.

Knöll B, Weinl C, Nordheim A, Bonhoeffer F (2007) Stripe assay to examine axonal guidance and cell migration. Nat Protoc 2:1216-1224.

Meiners S, Geller HM (1997) Long and short splice variants of human tenascin differentially regulate neurite outgrowth. Mol Cell Neurosci 10:100-116.

Mercado ML, Nur-e-Kamal A, Liu HY, Gross SR, Movahed R, Meiners S (2004) Neurite outgrowth by the alternatively spliced region of human tenascin-C is mediated by neuronal $\alpha 7 \beta 1$ integrin. J Neurosci 24:238-247.

Mombaerts P, Wang F, Dulac C, Chao SK, Nemes A, Mendelsohn M, Edmondson J, Axel R (1996) Visualizing an olfactory sensory map. Cell 87:675-686.

Nakamura K, Hioki H, Fujiyama F, Kaneko T (2005) Postnatal changes of vesicular glutamate transporter (VGluT)1 and VGluT2 immunoreactivities and their colocalization in the mouse forebrain. J Comp Neurol 492:263-288.

Ozaki S, Snider WD (1997) Initial trajectories of sensory axons toward laminar targets in the developing mouse spinal cord. J Comp Neurol 380:215-229.

Renzi MJ, Wexler TL, Raper JA (2000) Olfactory sensory axons expressing a dominant-negative semaphorin receptor enter the CNS early and overshoot their target. Neuron 28:437-447.

Saga Y, Yagi T, Ikawa Y, Sakakura T, Aizawa S (1992) Mice develop normally without tenascin. Genes Dev 6:1821-1831.

Schreyer DJ, Jones EG (1982) Growth and target finding by axons of the corticospinal tract in prenatal and postnatal rats. Neuroscience 7:1837-1853.

Schwarting GA, Raitcheva D, Crandall JE, Burkhardt C, Püschel AW (2004) Semaphorin 3A-mediated axon guidance regulates convergence and targeting of P2 odorant receptor axons. Eur J Neurosci 19:1800-1810.

Serizawa S, Miyamichi K, Takeuchi H, Yamagishi Y, Suzuki M, Sakano H (2006) A neuronal identity code for the odorant receptor-specific and activity-dependent axon sorting. Cell 127:1057-1069.

Shay EL, Greer CA, Treloar HB (2008) Dynamic expression patterns of 
ECM molecules in the developing mouse olfactory pathway. Dev Dyn 237:1837-1850.

Srinivasan J, Schachner M, Catterall WA (1998) Interaction of voltage-gated sodium channels with the extracellular matrix molecules tenascin- $\mathrm{C}$ and tenascin-R. Proc Natl Acad Sci U S A 95:15753-15757.

Steindler DA, Cooper NG, Faissner A, Schachner M (1989) Boundaries defined by addhesion molecules during development of the cerebral cortex: the J1/tenascin glycoprotein in the mouse somatosensory cortical barrel field. Dev Biol 131:243-260.

Stoykova A, Götz M, Gruss P, Price J (1997) Pax6-dependent regulation of adhesive patterning, R-cadherin expression and boundary formation in developing forebrain. Development 124:3765-3777.

Strekalova T, Sun M, Sibbe M, Evers M, Dityatev A, Gass P, Schachner M (2002) Fibronectin domains of extracellular matrix molecule tenascin-C modulate hippocampal learning and synaptic plasticity. Mol Cell Neurosci 21:173-187.

Strotmann J, Conzelmann S, Beck A, Feinstein P, Breer H, Mombaerts P (2000) Local permutations in the glomerular array of the mouse olfactory bulb. J Neurosci 20:6927-6938.

Taylor J, Pesheva P, Schachner M (1993) Influence of janusin and tenascin on growth cone behavior in vitro. J Neurosci Res 35:347-362.

Treloar H, Walters E, Margolis F, Key B (1996a) Olfactory glomeruli are innervated by more than one distinct subset of primary sensory olfactory neurons in mice. J Comp Neurol 367:550-562.

Treloar HB, Nurcombe V, Key B (1996b) Expression of extracellular matrix molecules in the embryonic rat olfactory pathway. J Neurobiol 31:41-55.

Treloar H, Tomasiewicz H, Magnuson T, Key B (1997) The central pathway of primary olfactory axons is abnormal in mice lacking the N-CAM-180 isoform. J Neurobiol 32:643-658.

Treloar HB, Purcell AL, Greer CA (1999) Glomerular formation in the developing rat olfactory bulb. J Comp Neurol 413:289-304.

von Holst A, Egbers U, Prochiantz A, Faissner A (2007) Neural stem/progenitor cells express 20 tenascin $\mathrm{C}$ isoforms that are differentially regulated by Pax6. J Biol Chem 282:9172-9181.

Wang F, Nemes A, Mendelsohn M, Axel R (1998) Odorant receptors govern the formation of a precise topographic map. Cell 93:47-60.

Wang G, Scott SA (2000) The "waiting period" of sensory and motor axons in early chick hindlimb: its role in axon pathfinding and neuronal maturation. J Neurosci 20:5358-5366.

Watanabe K, Tamamaki N, Furuta T, Ackerman SL, Ikenaka K, Ono K (2006) Dorsally derived netrin 1 provides an inhibitory cue and elaborates the "waiting period" for primary sensory axons in the developing spinal cord. Development 133:1379-1387. 